\title{
Ground state of magnetocrystals
}

\author{
N. Vandewalle $\odot$ and A. Wafflard \\ GRASP, Institute of Physics B5a, University of Liège, B4000 Liège, Belgium
}

(Received 20 June 2020; revised 25 February 2021; accepted 1 March 2021; published 15 March 2021)

\begin{abstract}
Neodyme spherical magnets are inexpensive objects that demonstrate how dipolar particles self-assemble into various structures ranging from $1 \mathrm{D}$ chains to $3 \mathrm{D}$ crystals. The dipole-dipole interactions confer the stability to these particular architectures. In the present paper, we explore ordered structures only, and we evidence that hybrid magnetocrystals, alternating hexagonal planes of antiparallel dipoles, have the lowest magnetic energy. This cohesion is the magnetic counterpart of the Madelung lattice energy found for ionic solids.
\end{abstract}

DOI: 10.1103/PhysRevE.103.032117

Let us imagine that you buy 60 magnetic balls at your favorite toy market. They stick together due to the magnetic attraction being much stronger than gravity forces. Billions of different configurations can be envisaged, but only a few of them are ordered structures. They already capture the complexity of assembling dipolar beads. Three typical examples, made of 60 spherical magnets, are shown in Fig. 1: (a) one simple loop, (b) one hexagonal plate with a vacancy in the center, and (c) one cubic lattice $3 \times 4 \times 5$. Finding the best arrangement for $N=60$ dipoles, which minimizes the magnetic energy of the system, is a challenge since it depends on all the positions and orientations of the dipoles.

Recently, magnetic chains [1,2], cubic structures [3-5], tubular [6], and planar structures [7] have been considered, using spherical permanent magnets. These experimental and numerical studies underlines the high complexity of magnetic structures. In chains, defects can be created in the perfect ordering of dipoles. These defects could act as magnetic monopoles [1]. By minimizing the energy of eight dipoles placed at the vertices of a cube, an infinite number of stables configurations have been evidenced, similar to Goldstone modes [3,4]. Moreover, these multipolar magnetic cubes may interact with extremely short-range interactions [5]. Hexagonal lattices have been also investigated, and the role played by lacunes has also been demonstrated [7]. Besides these fundamental questions, many applications can be found using dipolar particles, from magnetic colloids [8] to smart actuators [9] and microrobots [10].

In this paper, we will address the fundamental question of the magnetic cohesive energy $u$ of ordered 1D, 2D, and 3D structures being composed of many dipolar magnetic balls. These ordered structures are nicknamed magnetocrystals [11].

Let us note that the neodyme spheres behave as pointlike dipoles $[12,13]$ by assuming that they are uniformly magnetized. The interaction energy between two pointlike dipoles $\vec{m}_{\alpha}$ and $\vec{m}_{\beta}$ is given by

$$
U_{\alpha \beta}=\frac{\mu_{0}}{4 \pi}\left[\frac{\vec{m}_{\alpha} \cdot \vec{m}_{\beta}}{r_{\alpha \beta}^{3}}-3 \frac{\left(\vec{m}_{\alpha} \cdot \vec{r}_{\alpha \beta}\right)\left(\vec{m}_{\beta} \cdot \vec{r}_{\alpha \beta}\right)}{r_{\alpha \beta}^{5}}\right] .
$$

where $\vec{r}_{\alpha \beta}=\vec{r}_{\beta}-\vec{r}_{\alpha}$ is the vector linking particle centers $\alpha$ and $\beta$. Looking carefully at (1), one observes that, depending on relative positions and orientations, two dipoles can attract or repel each other. We will see in this paper that both effects are playing a role in ordered structures. In the following, we consider identical beads such that they have similar size and similar magnetization $\left(\left|\vec{m}_{\alpha}\right|=m\right)$. It is therefore possible to define a dimensionless magnetic cohesion per particle as

$$
u=\frac{2 \pi D^{3}}{N \mu_{0} m^{2}} \sum_{\alpha \neq \beta} U_{\alpha \beta},
$$

where $D$ is the sphere diameter. Since each dipole $\alpha$ has five degrees of freedom, being the positions $\left(x_{\alpha}, y_{\alpha}, z_{\alpha}\right)$ and angular orientations $\left(\theta_{\alpha}, \varphi_{\alpha}\right)$, minimizing the energy (2) in our example requires us to explore a complex configuration space with $5 \times 60$ degrees of freedom. Stochastic optimization algorithms can be used to find the ground states of Eq. (2), as proposed in Ref. [1]. However, this requires long computation times. In the example of 60 magnetic beads, as given above, one intuitively expects that compact and ordered structures will reach the lowest energy since particles are close together. But this is not the case. Simulations show that the hexagonal 2D structure of Fig. 1(b) has a lower cohesive energy than the cubic one [Fig. 1(c)]. Moreover, the cubic crystal is hard to obtain experimentally, reflecting the weak cohesive energy of this structure. This surprising result is due to the fact that the dipole orientations are highly relevant.

In this paper, we propose a different approach: we will assume a dipole ordering, compatible with experiments and observations, in order to calculate best arrangements. Calculations of magnetic cohesion $u$ will be obtained in the thermodynamic limit $N \rightarrow \infty$.

Let us start with the 1D chain. As discussed in Ref. [1], the ground state is obtained when all dipoles are parallel to the chain favoring attraction between spheres. Each dipole is interacting with its neighbors at distance $D, 2 D, 3 D$, and so on. The asymptotic value of the $1 \mathrm{D}$ ground state per dipole 

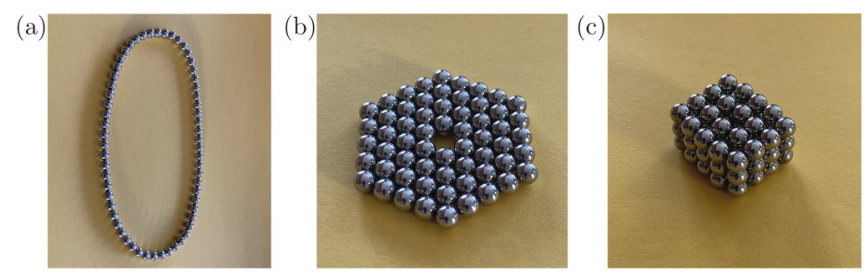

FIG. 1. Typical 1D, 2D, and 3D structures composed of 60 dipolar magnetic balls: (a) a chain forming a loop, (b) a hexagonal plate with a hole in the center, and (c) a cubic lattice.

can thus be evaluated by (2), which reduces to the series

$$
u_{1 \mathrm{~d}}=-2 \sum_{i=1}^{\infty} \frac{1}{i^{3}}=-2 \zeta(3),
$$

where all terms are attractive contributions to the magnetic cohesion. This sum reduces to the Riemann zeta function $\zeta$, giving an asymptotic value $u_{1 \mathrm{D}} \approx-2.4037$ for the dimensionless magnetic energy per particle [1]. Although magnetic chains and loops are basic systems, they can be assembled in a way to form defects in the dipole orientations such that monopole-like interactions are obtained, as demonstrated in Ref. [1]. It has also been demonstrated [2] that the elastic properties of rings like the one of Fig. 1(a) originate from the magnetic dipolar interactions.

Two-dimensional ordered structures can be assembled following two different ways. Chains with parallel dipoles are attracting if they form a hexagonal lattice. Antiparallel chains can also be assembled to form a square lattice. Figure 2 presents a sketch of both cases, emphasizing the dipole orientations in sublattice elements of different sizes.

Following the calculation method described in the Appendix and considering Eq. (2) for the hexagonal lattice, the cohesive energy reduces to

$$
u_{\text {hex }}=\sum_{(i, j)} \frac{i^{2}+8 i j-8 j^{2}}{8\left(i^{2}-i j+j^{2}\right)^{5 / 2}},
$$

(a)

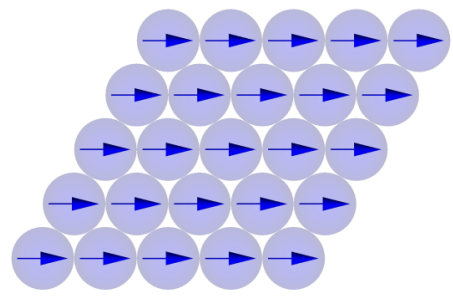

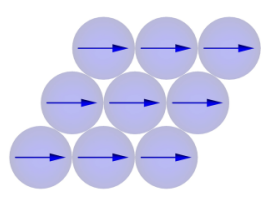

(b)
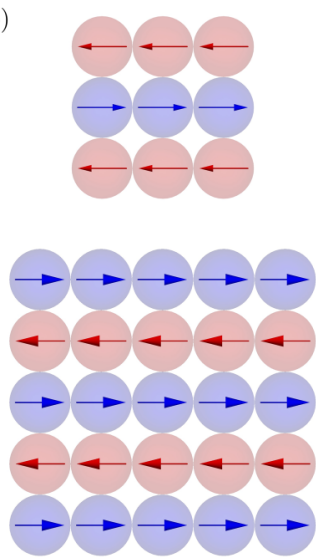

FIG. 2. Sketch of 2D sublattices with $3 \times 3(a=1)$ and $5 \times$ 5 dipoles $(a=2)$. Two types of arrangements can be created: (a) hexagonal lattice and (b) square lattice. Blue and red arrows emphasize (anti-)parallel dipole ordering in these structures.
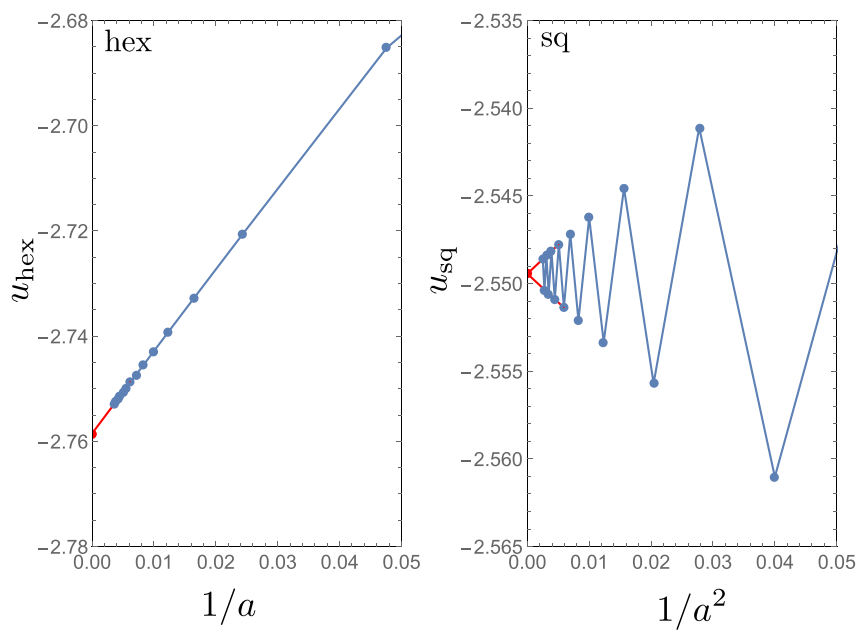

FIG. 3. For each 2D structure discussed in this paper, we present the evolution of the cohesive energy $u$ as a function of inverse crystal size $1 / a$. (Left) Hexagonal structure shows a $1 / a$ convergence towards -2.7585 . (Right) The square lattice shows however a $1 / a^{2}$ convergence towards -2.5494 for, respectively, odd and even $a$ values. Red lines illustrate the intercept with the vertical axis for the infinite system.

where $i$ and $j$ are lattice numbers for translational ordering, each particle being given by the couple $(i, j)$. The double sum runs over any particle $(i, j)$ of the lattice different from the regarded central particle $(i=0, j=0)$. The sums in (4) are dominated by attractive terms of nearest neighbors. No analytic solution can be obtained, and the series (4) should be evaluated numerically. For such a purpose, we consider lattices by running $i$ and $j$ in the growing interval $[-a,+a]$, i.e., by taking into account an increasing number $N=(2 a+1)^{2}$ of dipoles. Figure 3 (left) presents the numerical result as a function of $1 / a$. One assumes a linear behavior intercepting the vertical axis at $u_{\text {hex }}=-2.7585$ being the asymptotic value. This value is close to the one obtained earlier by Messina and coworkers in the case of planar [7] and tubular [6] arrangements. This value has also been theoretically approached [14].

The square lattice is obtained by using antiparallel dipoles from a line to another one, as illustrated in Fig. 2(b). The ground state is given by

$$
u_{\mathrm{sq}}=\sum_{(i, j)} \frac{(-1)^{j}\left(j^{2}-2 i^{2}\right)}{2\left(i^{2}+j^{2}\right)^{5 / 2}}
$$

in which the factor $(-1)^{i}$ induces major oscillations in the convergence of the series. Alternating attractive and repulsive magnetic forces can be viewed as the magnetic counterpart of the ionic solids, in which Coulomb attraction and repulsion alternate along lattice axes. The present calculation gives a kind of magnetic Madelung lattice energy. Figure 3 (right) presents $u_{\mathrm{sq}}$ as a function of $1 / a^{2}$. Oscillations are seen in $u_{\mathrm{sq}}$ as expected. However, a linear behavior is observed for respectively odd and even $a$ values. Both linear trends are converging towards a unique limit along the vertical axis. One obtains $u_{\mathrm{sq}}=-2.5494$. This value, reported to the first time to our knowledge, is higher than $u_{\text {hex }}$ whatever 
the value of $a$, meaning a weaker cohesion that could be observed in experiments. In particular, a planar square lattice seems harder to create than a planar hexagonal lattice. Comparing magnetostructures based on $u$ values is delicate since the mechanical stability is linked to the entire energy landscape around those minima. Cracks and particle reorganisations appear differently in square/hexagonal lattices such that situations are contrasted. Nevertheless, ranking lattices using $u$ allows one to draw a global trend.

Consider the packing fraction $\eta$ being in 2D the surface covered by magnetic beads over the lattice area. One obtains $\eta_{\text {hex }}=\frac{\pi}{2 \sqrt{3}}=0.9069$, being larger than $\eta_{\mathrm{sq}}=\frac{\pi}{4}=0.7854$. We can conclude that, in 2D, denser lattices of dipoles correspond also to higher cohesive crystals.

For 3D structures, one may consider the generalization of the cubic lattice by alternating antiparallel chains, as illustrated in Fig. 4(a). The cubic structure seems natural and is often used for advertising magnetic toys. However, creating a cubic lattice with neodyme magnets is quite tricky. For such a structure, one has

$$
u_{\mathrm{cub}}=\sum_{(i, j, k)} \frac{(-1)^{j+k}\left(-2 i^{2}+j^{2}+k^{2}\right)}{2\left(i^{2}+j^{2}+k^{2}\right)^{5 / 2}},
$$

where $k$ is the third lattice number. Figure 5 shows $u_{\text {cub }}$ as a function of $1 / a^{2}$. By increasing $a$, the balance between parallel and antiparallel rows has the same sign explaining a slow convergence instead of oscillations. Nevertheless, the intercept with the vertical axis gives $u_{\text {cub }}=-2.6767$. Although the 3D cubic lattice has a low cohesive value, it is still higher than hexagonal plates whatever their relative sizes $a$, as announced in the introduction.

Another common 3D structure is the fcc lattice which can be built from a stack of slightly shifted hexagonal layers. This is illustrated in Fig. 4(b). In order to obtain attraction between layers, dipoles should be oriented in the same way. This lattice possesses the highest packing fraction value ever known, $\eta_{\mathrm{fcc}}=\frac{\pi}{3 \sqrt{2}} \approx 0.7405$ [15], but this structure has a weak magnetic cohesion. Indeed, one has

$$
u_{\mathrm{fcc}}=\sum_{(i, j, k)} \frac{-8 i^{2}-8 i(j+k)+(j-k)^{2}}{8\left[i^{2}+i(j+k)+j^{2}+j k+k^{2}\right]^{5 / 2}},
$$

which tends to $u_{\mathrm{fcc}}=-0.5763$ in the thermodynamic limit. This magnetic cohesion is higher than the reference cohesion of a single pair of dipoles $(u=-1)$. As a consequence, this structure is hard to create experimentally, and any mechanical stress destroys this extremely fragile magnetostructure.

A third structure can be created by stacking hexagonal planes placed in antiparallel situation. The so-called hybrid structure is illustrated in Fig. 4(c). Its cohesive energy is given by

$$
u_{\mathrm{hyb}}=\sum_{(i, j, k)} \frac{(-1)^{k}\left(i^{2}+8 i j-8 j^{2}+4 k^{2}\right)}{8\left(i^{2}-i j+j^{2}+k^{2}\right)^{5 / 2}}
$$

where the $(-1)^{k}$ factor emphasizes the alternating stack of antiparallel dipoles. Figure 5 shows the convergence as a (a)
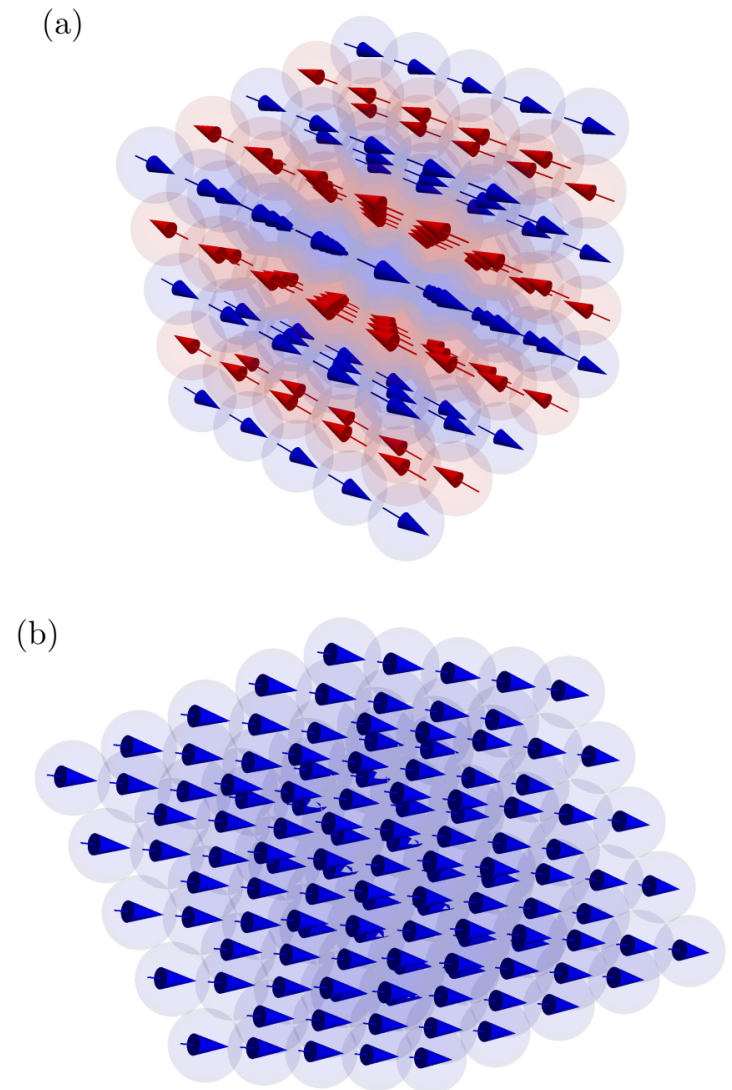

(c)

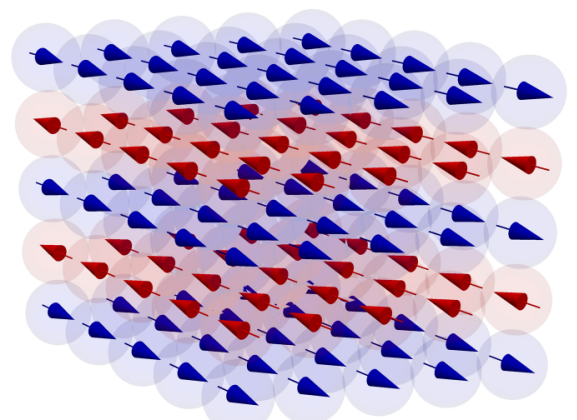

FIG. 4. Sketch of 3D lattices with $5 \times 5 \times 5$ dipoles. Three different arrangements can be created: (a) cubic lattice composed of alternating antiparallel chains of dipoles, (b) fcc lattice corresponding to the compact arrangement superposing similar hexagonal layers being slightly shifted, and (c) hybrid lattice composed of antiparallel hexagonal planes. Blue and red arrows emphasize (anti-)parallel dipole ordering in the structure.

function $1 / a$ such that $u_{\text {hyb }}=-2.8712$ is obtained. Therefore, hybrid lattices have the lowest cohesive magnetic energy known. They should be considered the most stable magnetostructures. Recent experiments have been performed with magnetocrystals showing indeed that the elastic response and strength of hybrid lattices are enhanced [11] compared to other magnetocrystals.

We have calculated above the cohesive energy of 2D and 3D magnetocrystals. The results are summarized in Table I. The hybrid lattice seems to have the lowest cohesion energy 

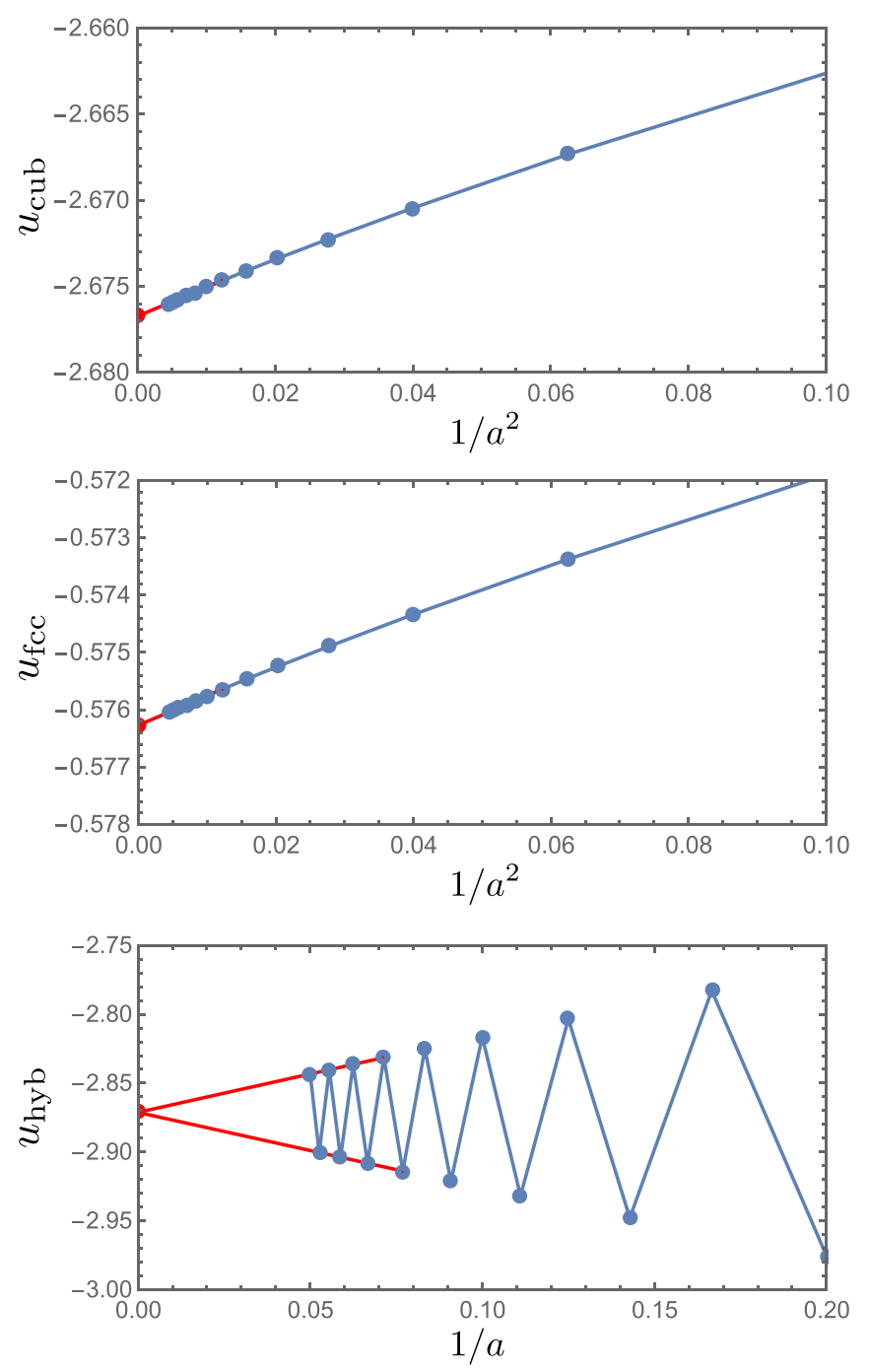

FIG. 5. For each 3D case discussed in this paper, we present the evolution of the cohesive energy $u$ as a function of the inverse crystal size $1 / a$ or $1 / a^{2}$. Red lines illustrate the intercept with the vertical axis for the infinite system.

for a packing fraction $\eta_{\text {hyb }}=\frac{\pi}{3 \sqrt{3}}$, although this structure is far from being the most densest one.

Our results about the cohesive energy of structures made of magnetic entities may find some applications in magnetic colloids $[8,16]$. Indeed, magnetic colloids can be synthesized

TABLE I. The various structures discussed in this paper and their ground state $u$, as well as their packing fraction $\eta$. References to earlier calculations are also given.

\begin{tabular}{lcccc}
\hline \hline$d$ & Magnetostructure & $u$ & $\eta$ & References \\
\hline 1 & Chain & -2.4037 & 1 & {$[1,2]$} \\
2 & Square & -2.5494 & 0.7854 & This work \\
2 & Hexagonal & -2.7585 & 0.9069 & {$[6,7,14]$} \\
3 & Cubic & -2.6767 & 0.5236 & This work \\
3 & fcc & -0.5763 & 0.7405 & This work \\
3 & Hybrid & -2.8712 & 0.6046 & This work \\
\hline \hline
\end{tabular}

in a variety of shapes $[17,18]$, and the possibility of remotely controlling these soft matter entities via external fields makes them ideal for producing tiny ordered structures, which, for example, could be useful for pharmaceutical applications [8]. The 2D crystalization and more specifically the formation of hexagonal 2D ribbons [19] has been proposed to better capture the formation of colloidal structures under a constant magnetic field.

Micrometer- up to millimeter-sized magnetic particles can also be used for the microfabrication of 3D electronic devices exploiting the magnetic interactions for self-assembling specific and functional [20,21]. Self-assembly using dipolar objects of various shapes can help scientists to microfabricate 2D and 3d metamaterials as demonstrated in Ref. [22]. Cubic lattices have been grown by self-assembly in that case. It has been also shown that microstructures can be self-assembled [23] along liquid interfaces when the balance is reached between magnetic and capillary interactions. Those particles can form 2D hexagonal lattices that can be actuated for mimicking complex microorganisms [10].

Dipolar interactions between particles are also used to induce cohesion in random packing experiments [24] and more recently to excite systems like granular gases in order to control the agitation, i.e., the injection of mechanical energy, allowing for an extensive experimental study for the equation of state in these dissipative systems [25].

A last application is the development of magnetorheological elastomer by placing an array of magnetic particles in an elastic matrix [26]. Using an external field orienting the dipoles, the magnetic particles can be used for bending or stretching these elastic composites creating smart actuators $[9,27]$. The way particles are arranged in the matrix is relevant for creating different behaviors [28] and instabilities [9].

In summary, the study of ordered structures made of dipole magnets reveal that the hardest known structure is the hybrid magnetocrystal, being made of a stack of antiparallel hexagonal plates. We show that it is characterized by a Madelung-like lattice energy. This may find some applications in soft matter where magnetic particles are used for self-assembly, colloidal structures, microrobots, and actuators.

\section{ACKNOWLEDGMENTS}

This work is financially supported by the University of Liège through the CESAM Research Unit.

\section{APPENDIX: CALCULATION DETAILS}

This section gives more details on the calculation method. This method is illustrated with the specific cases of a 1D chain, 2D square lattice, and 3D cubic lattice.

Since we evaluate the magnetic cohesion $u$ in the thermodynamic limit, we consider that instead of using all pairs $(\alpha, \beta)$ of dipoles, we look only at the central dipole $(\alpha=0)$ and the sum runs over all dipoles $\beta \neq 0$. Equation (2) becomes

$$
u=\frac{2 \pi}{\mu_{0}} \frac{D^{3}}{m^{2}} \sum_{\beta \neq 0} U_{0 \beta} .
$$

For a 1D chain of dipoles, one has a single lattice number $\beta=i$ that can be used to label dipoles from $-\infty$ to $\infty$. Since 
the dipoles are parallel to the chain, one has $\vec{m}_{0} \cdot \vec{m}_{\beta}=m^{2}$ and $\vec{m}_{0} \cdot \vec{r}_{0 \beta}=\vec{m}_{\beta} \cdot \vec{r}_{0 \beta}=m i D$. Considering Eq. (1) and Eq. (A1), one obtains

$$
u_{1 \mathrm{D}}=\frac{1}{2} \sum_{i \neq 0}\left(\frac{1-3}{i^{3}}\right)
$$

which reduces to Eq. (3), giving the expected result.

For a square lattice, with alternating dipole orientations, we consider the dipole placed at the center of the $(2 a+1) \times$ $(2 a+1)$ square. Two lattice numbers $i$ and $j$ are needed to run over the dipoles $\beta$. Dipoles are parallel to the axis defined by lattice number $i$. One has therefore $\vec{m}_{0} \cdot \vec{m}_{\beta}=$ $(-1)^{j} m^{2}$, the factor $(-1)^{j}$ being used to model the alternating orientations of the dipoles. Moreover the scalar products in Eq. (1) become $\left(\vec{m}_{0} \cdot \vec{r}_{0 \beta}\right)\left(\vec{m}_{\beta} \cdot \vec{r}_{0 \beta}\right)=(-1)^{j} m^{2} i^{2} D^{2}$. Finally, one has

$$
u_{\mathrm{sq}}=\frac{1}{2} \sum_{(i, j)}(-1)^{j}\left[\frac{1-3 \frac{i^{2}}{\left(i^{2}+j^{2}\right)}}{\left(i^{2}+j^{2}\right)^{3 / 2}}\right],
$$

which reduces to Eq. (5), which we numerically evaluate in the main text for large lattices.

For the cubic 3D lattice, a third lattice number $k$ should be added. Dipole orientations alternate over $j$ and $k$ lattice numbers. The evaluation of Eq. (A1) gives

$$
u_{\mathrm{cub}}=\frac{1}{2} \sum_{(i, j, k)}(-1)^{j+k}\left[\frac{1-3 \frac{i^{2}}{\left(i^{2}+j^{2}+k^{2}\right)}}{\left(i^{2}+j^{2}+k^{2}\right)^{3 / 2}}\right],
$$

which reduces to Eq. (6), as expected. The magnetic cohesion of the other lattices was evaluated following the same method.
[1] N. Vandewalle and S. Dorbolo, New J. Phys. 16, 013050 (2014).

[2] D. Vella, E.du Pontavice, C. L. Hall, and A. Goriely, Proc. Roy. Soc. A 470, 2162 (2014).

[3] S. Borgers, S. Völkel, W. Schöpf, and I. Rehberg, Am. J. Phys. 86, 460 (2018).

[4] J. Schönke, T. M. Schneider, and I. Rehberg, Phys. Rev. B 91, 020410(R) (2015).

[5] S. Hartung, F. Sommer, S. Völkel, J. Schönke, and I. Rehberg, Phys. Rev. B 98, 214424 (2018).

[6] R. Messina, L. A. Khalil, and I. Stanković, Phys. Rev. E 89, 011202(R) (2014).

[7] R. Messina and I. Stankovic, Europhys. Lett. 110, 46003 (2015).

[8] P. Tierno, Phys. Chem. Chem. Phys. 16, 23515 (2014).

[9] M. Poty, F. Weyer, G. Grosjean, G. Lumay, and N. Vandewalle, Eur. Phys. J. E 40, 29 (2017).

[10] Y. Collard, G. Grosjean, and N. Vandewalle, Commun. Phys. 3, 112 (2020).

[11] S. Hidalgo-Caballero, Y. Y. Escobar-Ortegaa, R. I. BecerraDeana, J. M. Salazar, F. Pacheco-Vázquez, J. Magn. Magn. Mater. 479, 149 (2019).

[12] J. D. Jackson, Classical Electrodynamics, 3rd ed. (Wiley, New York, 1998).

[13] R. E. Rosensweig, Ferrohydrodynamics (Dover Publications, New York, 1997).

[14] A. Grzybowski and A. Brodka, Mol. Phys. 101, 1079 (2003).
[15] T. Aste and D. Weaire, The Pursuit of Perfect Packing, 2nd ed. (Taylor \& Francis, Boca Raton, FL, 2008).

[16] P. G. de Gennes and P. A. Pincus, Phys. Kondens. Mater. 11, 189 (1970).

[17] M. Nakade, T. Ikeda, and M. Ogawa, J. Mater. Sci. 42, 4815 (2007).

[18] L. Rossi, S. Sacanna, W. T. M. Irvine, P. M. Chaikin, D. J. Pine, and A. P. Philipse, Soft Matter. 7, 4139 (2011).

[19] A. Darras, J. Fiscina, M. Pakpour, N. Vandewalle, and G. Lumay, Eur. Phys. J. E 39, 47 (2016).

[20] G. M. Whitesides and B. Grzybowski, Science 295, 2418 (2002).

[21] J. A. Pelesko, Self-Assembly (Chapmann \& Hall, Boca Raton, FL, 2007).

[22] L. Abelmann, T. A. G. Hageman, P. A. Löthman, M. Mastrangeli, M. C. Ewenspoek, Sci. Adv. 6, eabea2007 (2020).

[23] G. Grosjean, M. Hubert, and N. Vandewalle, Adv. Colloid Interface Sci. 255, 84 (2018).

[24] G. Lumay and N. Vandewalle, New J. Phys. 9, 406 (2007).

[25] E. Falcon, J.-C. Bacri, and C. Laroche, Europhys. Lett. 103, 64004 (2013).

[26] Ubaidillah, J. Sutrisno, A. Purwanto, and S. A. Mazlan, Adv. Eng. Mat. 17, 563 (2015).

[27] T. Xu, J. Zhang, M. Salehizadeh, O. Onaizah, and E. Diller, Sci. Robot. 4, eaav4494 (2019).

[28] A. Goshkoderia, V. Chen, J. Li, A. Juhl, P. Buskohl, and S. Rudykh, Phys. Rev. Lett. 124, 158002 (2020). 